\title{
Risk of diabetes after gestational diabetes and preeclampsia. A registry-based study of 230,000 women in Norway
}

\author{
Anders Engeland · Tone Bjørge • Anne Kjersti Daltveit • \\ Svetlana Skurtveit · Siri Vangen · Stein Emil Vollset • \\ Kari Furu
}

Received: 5 August 2010/Accepted: 18 November 2010/Published online: 6 February 2011

(C) The Author(s) 2011. This article is published with open access at Springerlink.com

\begin{abstract}
This study aimed to use a population-based Prescription Database to explore later development of diabetes in women registered with gestational diabetes mellitus (GDM) and/or preeclampsia in the Medical Birth Registry of Norway (MBRN) during 2004-8. We used two nationwide Norwegian registries, the Norwegian Prescription Database and the MBRN, to explore the onset of later diabetes after pregnancy complications, indicated by receiving prescriptions of drugs used to treat diabetes, in 230,000 women
\end{abstract}

A. Engeland $(\bowtie) \cdot$ S. Skurtveit $\cdot$ K. Furu

Department of Pharmacoepidemiology, Division of

Epidemiology, Norwegian Institute of Public Health, Nydalen,

Oslo, Norway

e-mail: anders.engeland@isf.uib.no

T. Bjørge · A. K. Daltveit · S. E. Vollset

Medical Birth Registry of Norway, Division of Epidemiology, Norwegian Institute of Public Health, Kalfarveien 31, Bergen, Norway

A. Engeland - T. Bjørge - A. K. Daltveit - S. E. Vollset Department of Public Health and Primary Health Care,

University of Bergen, Bergen, Norway

\section{S. Skurtveit}

Norwegian Centre for Addictive Research, University of Oslo, Oslo, Norway

S. Vangen

National Resource Centre for Women's Health, Oslo University

Hospital, Unit Rikshospitalet, Oslo, Norway

S. Vangen

Department of Chronic Diseases, Division of Epidemiology,

Norwegian Institute of Public Health, Oslo, Norway

K. Furu

Department of Pharmacy, University of Troms $\varnothing$, Troms $\varnothing$,

Norway giving birth in 2004-8. The mean follow-up of the study cohort was 3.7 years. Five years after pregnancy, about 19 and $2 \%$ of women with GDM and preeclampsia, respectively, received drugs used to treat diabetes, compared to $0.5 \%$ of those without these complications. The risk of being dispensed drugs used to treat diabetes within the first years after pregnancy was estimated to be 41 times (95\% CI: 35-47) and 3.0 times (95\% CI: 2.4-3.6) higher in women with GDM and preeclampsia, respectively, compared to women without these pregnancy complications. Women with pregnancies complicated with preeclampsia or GDM had an increased risk of later diabetes, especially those having GDM. If the increase in frequency of GDM observed in MBRN in recent years is real, a further increase in diabetic women can be expected.

Keywords Cohort - Diabetes mellitus - Drug prescriptions $\cdot$ Population-based registries

$\begin{array}{ll}\text { Abbreviations } \\ \text { ATC } & \text { Anatomical therapeutic chemical } \\ \text { CI } & \text { Confidence interval } \\ \text { GDM } & \text { Gestational diabetes mellitus } \\ \text { ICD } & \text { International Classification of Diseases } \\ \text { MBRN } & \text { Medical Birth Registry of Norway } \\ \text { NorPD } & \text { Norwegian Prescription Database } \\ \text { PCOS } & \text { Polycystic ovary syndrome } \\ \text { RR } & \text { Relative risk }\end{array}$

\section{Introduction}

Various forms of diabetes are a common and increasing health problem in many parts of the world. Worldwide, the number of adults above 20 years with diabetes has been 
estimated to 171 million in year 2000 and 366 million in 2030 [1]. Deaths attributable to diabetes in year 2000 have been estimated to 2.9 million worldwide [2].

Gestational diabetes mellitus (GDM) develops during pregnancy and usually disappears thereafter [3, 4]. In the US, GDM affects about $4 \%$ of pregnant women [5]. In Norway, the incidence of GDM is relatively low. In 2008, GDM appeared in about $1.3 \%$ of the pregnancies, being about doubled during the last decade, according to statistics from Medical Birth Registry of Norway (MBRN) [6]. Maternal diabetes type 1 and 2 appeared in 0.4 and $0.3 \%$ of the pregnancies in 2008 , respectively.

Several studies have linked GDM to increased risk of later diabetes [7-10], especially type 2 diabetes. Another disorder during pregnancy, preeclampsia, has been linked to GDM [11, 12] and also to later diabetes [13, 14]. Preeclampsia is, in Norway, much more common than GDM; preeclampsia was reported in about $3.6 \%$ of the pregnancies in 2008 [6].

The establishment of the Norwegian Prescription Database (NorPD) in 2004 made it possible to link data on maternal diseases during pregnancy from the MBRN with the maternal use of medication [15]. This study aimed to use the population-based NorPD to explore later development of diabetes in women registered with GDM and/or preeclampsia in MBRN during 2004-8.

\section{Materials and methods}

\section{Data sources}

Norwegian prescription database [16] is a research database which captures all dispensed prescriptions in Norway from January 1st, 2004, and covers the entire population of Norway (4.9 million). NorPD contains information on all prescribed drugs, reimbursed or not, dispensed at pharmacies to individual patients treated in ambulatory care. Data on use in institutionalized patients in nursing homes and hospitals are also collected, but these figures are only registered at an institutional and not at the individual level. Therefore, drugs dispensed to institutions are not included in our study. For each prescription, the sex and age of the patient, demographic information, dispensing date, and detailed drug information are registered. Until March 2009, the indication for prescribing was not recorded. Classification of drugs is based on the Anatomical Therapeutic Chemical (ATC) classification system [17].

Medical Birth Registry of Norway is a population based registry containing information on all births in Norway since 1967 (nearly 2.5 million births) [18]. MBRN is based on compulsory notification of every birth or late abortion from 12 completed weeks of gestation onwards, and includes identification on the parents by their personal identity numbers, demographic information of the parents, the mother's diseases before and during pregnancy, complications during pregnancy and delivery, length of pregnancy as well as information on the infant, including birth defects and other perinatal problems [18].

Maternal diseases, before and during pregnancy are coded by the staff at the MBRN using International Classification of Diseases (ICD) revision 10. In this study, we used variables for preeclampsia and GDM as defined by MBRN. The coding of GDM and diabetes in general in MBRN is described by Stene et al. [19]. The diagnostic criteria for preeclampsia in Norway have been blood pressure $\geq 140 / 90$ after 20 weeks of gestation, combined with proteinuria $\geq 0.3 \mathrm{~g} / 24 \mathrm{~h})(\geq+1$ dipsticks) on at least two occasions [20]. From 1999 onwards, a variable indicating whether preeclampsia was diagnosed before week 34 of gestation and a variable indicating whether the preeclampsia was mild or severe have been included in MBRN. In this study, these two variables were combined, preeclampsia diagnosed before week 34 was regarded as severe.

\section{Study subjects}

Data in MBRN, including eventual date of death/emigration for mother and child, were linked to NorPD using the unique 11-digit identification number, assigned to all individuals living in Norway after 1960. In our study, women with pregnancies (excluding abortions) registered in MBRN during 2004-8, lasting more than 22 weeks were included $(n=231,668$ pregnancies when counting each women's first pregnancy in the period). Only each woman's first pregnancy in the study period was included in our study. After exclusion of women with diabetes, polycystic ovary syndrome (PCOS) or chronic hypertension before pregnancy according to MBRN, the remaining number of women was 228,116. According to NorPD, 1,126 women (i.e. $0.5 \%$ of the pregnant women) received drugs used to treat diabetes (ATC code A10) prior to pregnancy (possible pre-existing diabetes mellitus). In addition, among women not having GDM, 47 women used insulin during pregnancy according to MBRN and 111 women not having registered GDM received drugs used to treat diabetes during pregnancy according to NorPD. After excluding all these women, 226,832 women remained (Fig. 1).

These women were followed with regard to prescriptions from the latest of January 1, 2004 and the date of giving birth, until prescription of the drugs in question, emigration or death or December 31, 2009, whichever occurred first. 


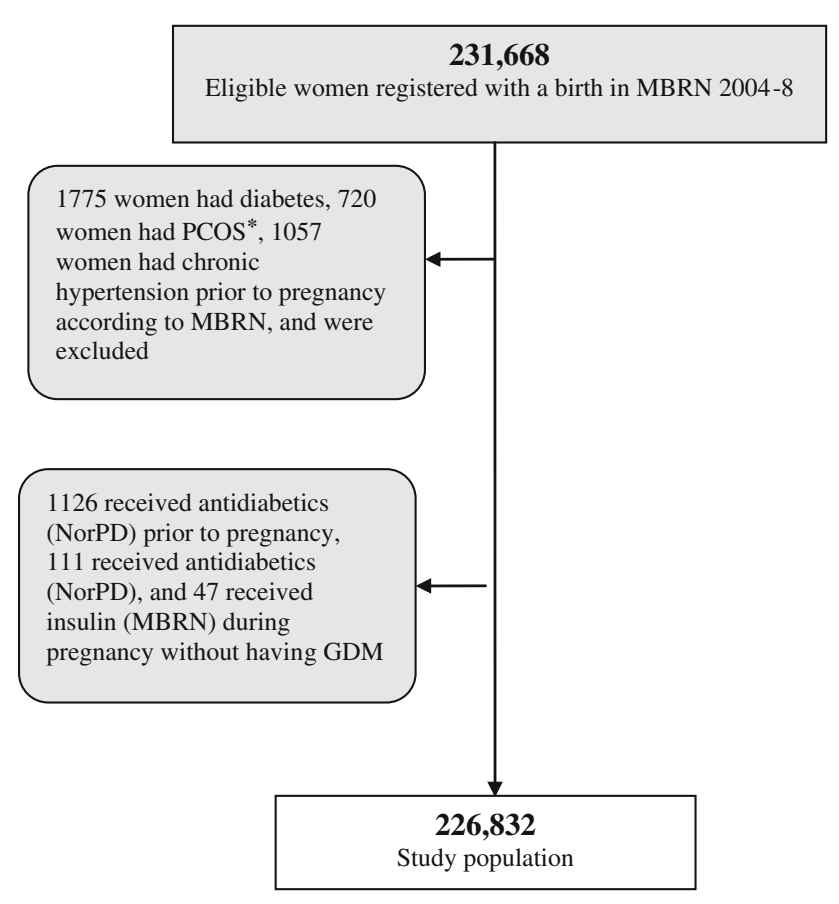

Fig. 1 Flow chart of the study population. Births registered in the Medical Birth Registry of Norway (MBRN) 2004-8 linked to the nationwide Norwegian Prescription Database (NorPD) 2004-9. *PCOS- Polycystic ovary syndrome

\section{Methods}

Prescriptions of drugs used to treat diabetes (ATC code A10) were used as proxy for diabetes. Prescriptions were divided into insulin (A10A: fast-acting, intermediate acting, intermediate-acting combined with fast-acting, longacting [17]) and oral antidiabetics (A10B: biquanides, sulfonamides, alpha glucosidase inhibitors, thiazolidinediones, meglitinides [17]). Those receiving drugs used to treat diabetes were divided into three groups; those receiving insulin only, those receiving oral antidiabetics only and those receiving both insulin and oral antidiabetics. Prescriptions during subsequent pregnancies were excluded.

The time from giving birth to the date of receiving drugs or the censoring date was analysed with the use of the Kaplan-Meier method and Cox proportional hazards models, with adjustment for maternal age, tested as categorical ( $<20,20-29,30-39$ and $\geq 40$ years) but included as continuous, and for parity (first pregnancy, second pregnancy, third pregnancy or more).

Hazard ratios, in this paper denoted relative risks (RRs), of receiving dispensed drugs used to treat diabetes were estimated in multivariate Cox proportional hazards models. Using maternal age as continuous variable in the models gave similar results. Since women with GDM may be screened for diabetes at some time postpartum, analyses were also performed excluding the first 6 months after giving birth.

The analysis were repeated using prescription of insulin (ATC code A10A) and oral antidiabetics (A10B), respectively, instead of the whole group of drugs used to treat diabetes (A10).

Data handling and analyses were performed using Stata and SPSS [21, 22].

\section{Results}

Altogether, 2,198 (1.0\%) of the included pregnancies were complicated with GDM and 8,832 (3.9\%) with preeclampsia, according to MBRN (Table 1). In pregnancies with GDM (mean maternal age 32 years), the mothers were older than in pregnancies without GDM (mean maternal age 30 years). Among mothers with GDM, 176 (8.0\%) had preeclampsia as well. After a mean follow-up of 3.7 years (range: 0-6 years after birth), 1,193 (0.5\%) of all mothers received prescriptions of drugs used to treat diabetes (ATC: A10); 0.4 and $14 \%$ of those without and with GDM and 0.5 and $1.6 \%$ of those without and with preeclampsia, respectively. Among those receiving drugs, 83\% received biguanides (A10BA), 21\% received intermediate-acting (A10AC) and 20\% received fast-acting insulins and analogues for injection (A10AB). Overall, about $0.5 \%$ of women without GDM and preeclampsia received a prescription of drugs used to treat diabetes during the first 5 years after giving birth.

Overall, about $19 \%$ of the mothers with GDM without preeclampsia received a prescription of drugs used to treat diabetes (insulin and/or oral antidiabetics) during the first 5 years after giving birth (Fig. 2). The number of women included in the calculations of frequency of receiving antidiabetics is shown in Table 2. These women had about 41 times higher risk of receiving drugs used to treat diabetes than those without GDM or preeclampsia (Table 3). About $2 \%$ of the mothers with preeclampsia without GDM received a prescription of drugs used to treat diabetes (insulin and/or oral antidiabetics) during the first 5 years after giving birth. These women had 3.0 times higher risk of receiving drugs used to treat diabetes than those without GDM or preeclampsia. Women with severe preeclampsia (33\% of the preeclamptic pregnancies) had a higher risk of receiving prescription of drugs used to treat diabetes than other women with preeclampsia (RR 1.6, 95\% CI 1.2-2.1).

Analyses excluding the first 6 months after giving birth, gave similar results.

We also studied the risks of receiving insulin or oral antidiabetics separately:

Compared with those receiving drugs used to treat diabetes in general, the group receiving insulin only had a 
Table 1 Characteristics of the study population
Data from pregnancies in 2004-8 ( $\mathrm{n}=226,832)$, Medical Birth Registry of Norway (MBRN) and from the Norwegian Prescription Database (NorPD)

GDM Gestational diabetes mellitus

\begin{tabular}{|c|c|c|c|c|}
\hline & \multicolumn{2}{|c|}{ Pregnancies without GDM } & \multicolumn{2}{|c|}{ Pregnancies with GDM } \\
\hline & $\begin{array}{l}\text { Total no. of } \\
\text { women }\end{array}$ & $\%$ & $\begin{array}{l}\text { Total no. of } \\
\text { women }\end{array}$ & $\%$ \\
\hline \multicolumn{5}{|l|}{ Year of childbirth } \\
\hline 2004 & 54,659 & 24.3 & 472 & 24.3 \\
\hline 2005 & 52,096 & 23.2 & 433 & 23.2 \\
\hline 2006 & 45,259 & 20.1 & 478 & 20.2 \\
\hline 2007 & 37,651 & 16.8 & 388 & 16.8 \\
\hline 2008 & 34,969 & 15.6 & 427 & 15.6 \\
\hline \multicolumn{5}{|l|}{ Maternal age (years) } \\
\hline$<20$ & 6,063 & 2.7 & 21 & 1.0 \\
\hline $20-29$ & 105,675 & 47.0 & 750 & 34.1 \\
\hline $30-39$ & 106,921 & 47.6 & 1,264 & 57.5 \\
\hline $40+$ & 5,975 & 2.7 & 163 & 7.4 \\
\hline \multicolumn{5}{|l|}{ Length of pregnancy (weeks) } \\
\hline $22-24$ & 351 & 0.2 & 1 & 0.0 \\
\hline $25-36$ & 13,902 & 6.2 & 238 & 10.8 \\
\hline $37-39$ & 89,555 & 39.9 & 1,198 & 54.4 \\
\hline $40-41$ & 103,994 & 46.3 & 712 & 32.4 \\
\hline $42+$ & 16,832 & 7.5 & 52 & 2.4 \\
\hline \multicolumn{5}{|l|}{ Preeclampsia } \\
\hline No & 215,988 & 96.2 & 2,022 & 92.0 \\
\hline Yes & 8,646 & 3.8 & 176 & 8.0 \\
\hline \multicolumn{5}{|l|}{ Condition } \\
\hline Stillbirth & 890 & 0.4 & 4 & 0.2 \\
\hline Livebirth & 223,744 & 99.6 & 2,194 & 99.8 \\
\hline \multicolumn{5}{|l|}{ Medication after pregnancy (2004-9) } \\
\hline Drugs used to treat diabetes (A10) & 891 & 0.4 & 302 & 13.7 \\
\hline \multicolumn{5}{|l|}{ Of these } \\
\hline Only insulin (A10A) & 122 & 13.1 & 106 & 34.4 \\
\hline Only oral antidiabetics (A10B) & 772 & 82.9 & 144 & 46.8 \\
\hline Both insulin and oral antidiabetics & 37 & 4.0 & 58 & 18.8 \\
\hline Total & 224,634 & 100 & 2,198 & 100 \\
\hline
\end{tabular}

risk of receiving oral antidiabetics than those without GDM or preeclampsia. About $2 \%$ of the mothers with preeclampsia without GDM received a prescription of oral antidiabetics during the first 5 years after giving birth. These women had 3.0 times (95\% CI 2.4-3.8) higher risk of receiving oral antidiabetics than those without GDM or preeclampsia.

\section{Discussion}

This study included almost 230,000 pregnant women giving birth during 2004-8. Nearly 2,200 women developed GDM. After a mean follow-up of 3.7 years, almost 1,200 women were dispensed drugs used to treat diabetes (insulin and/or oral antidiabetics). The probability of being dispensed drugs used to treat diabetes was estimated to be 41 


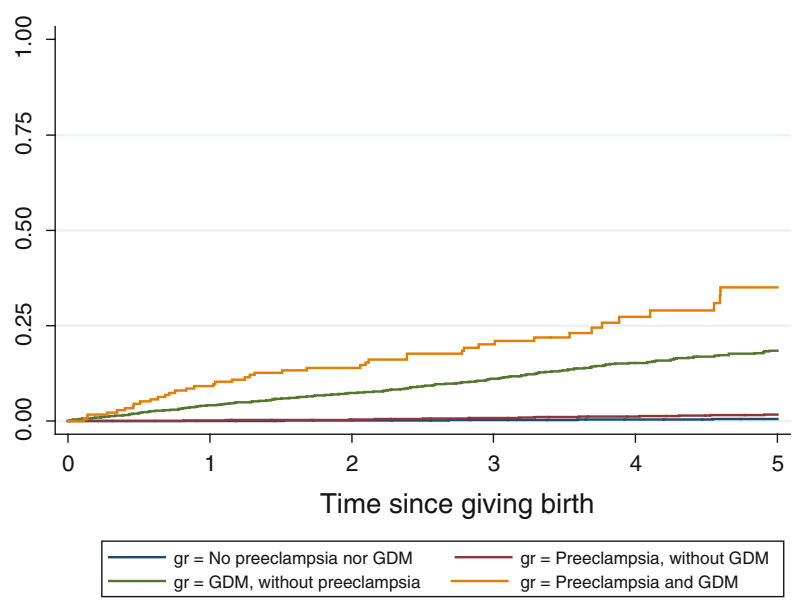

Fig. 2 Kaplan-Meier graph of estimated overall proportion of mothers receiving drugs used to treat diabetes (ATC-code A10) by time since giving birth, by disease status (GDM and/or preeclampsia). The estimates are based on data from women giving birth 2004-8 $(\mathrm{n}=226,832)$

times and 3 times higher in women with GDM and preeclampsia, respectively, compared to women without these pregnancy complications.
The major strength of our study was the use of two nationwide registries, the MBRN and the NorPD, and the unique 11-digit personal identity number, which ensured valid data linkage between the two registries. Other advantages were the large sample size, a prospective design and that the NorPD includes prescriptions from all practicing physicians prescribing to patients living outside institutions in Norway, both general practitioners and specialists. Further, the study covered all births in the Norwegian population (about 4.9 million).

Recently, the diabetic diagnoses in MBRN have been compared with NorPD [23]. The sensitivity, i.e. the proportion of women indicated to be diabetic in NorPD that was registered as diabetic in MBRN, was found to be $72 \%$. In our study, 1,775 mothers were excluded due to previous diagnoses of diabetes. Assuming a sensitivity of $72 \%$, another 700 mothers should have been excluded. Based on information from NorPD, we excluded additionally 1,126 women that received antidiabetics prior to pregnancy. In the previous study $3 \%$ of those with GDM received drugs used to treat diabetes during the 3 months prior to pregnancy. Even if $3 \%$ of mothers registered with GDM was diabetic before pregnancy, and hence did not have GDM,

Table 2 Number of women without previously received prescriptions of antidiabetics by years since giving birth

\begin{tabular}{|c|c|c|c|c|c|c|}
\hline & \multicolumn{6}{|c|}{ Years since giving birth } \\
\hline & 0 & 1 & 2 & 3 & 4 & 5 \\
\hline \multicolumn{7}{|l|}{ Group } \\
\hline No preeclampsia nor GDM & 215,988 & 215,202 & 181,318 & 145,095 & 101,771 & 51,995 \\
\hline Preeclampsia (without GDM) & 8,646 & 8,615 & 7,163 & 5,630 & 3,874 & 1,919 \\
\hline GDM (without preeclampsia) & 2,022 & 2,012 & 1,615 & 1,258 & 831 & 433 \\
\hline Preeclampsia and GDM & 176 & 175 & 138 & 109 & 67 & 34 \\
\hline Total & 226,832 & 226,004 & 190,234 & 152,092 & 106,543 & 54,381 \\
\hline
\end{tabular}

GDM Gestational diabetes mellitus

Table 3 Relative risk of getting dispensed drugs used to treat diabetes (insulin and/or oral antidiabetics), with 95\% confidence intervals (95\% $\mathrm{CI})$, obtained in Cox regression analysis, adjusted for maternal age and parity

\begin{tabular}{|c|c|c|c|c|c|c|c|c|c|}
\hline & \multirow[t]{2}{*}{$\%$} & \multicolumn{2}{|c|}{$\begin{array}{l}\text { Drugs used to treat } \\
\text { diabetes }\end{array}$} & \multicolumn{2}{|c|}{$\begin{array}{l}\text { Both insulin and oral } \\
\text { antidiabetics* }\end{array}$} & \multicolumn{2}{|c|}{ Oral antidiabetics only } & \multicolumn{2}{|l|}{ Insulin only } \\
\hline & & Relative risk & $95 \% \mathrm{CI}$ & Relative risk & $95 \% \mathrm{CI}$ & Relative risk & $95 \% \mathrm{CI}$ & Relative risk & $95 \% \mathrm{CI}$ \\
\hline \multicolumn{10}{|l|}{ Group } \\
\hline No preeclampsia nor GDM & 95.2 & 1.0 & Referent & 1.0 & Referent & 1.0 & Referent & 1.0 & Referent \\
\hline Preeclampsia (without GDM) & 3.8 & 3.0 & $2.4-3.6$ & 4.2 & $1.6-11$ & 3.0 & $2.4-3.7$ & 2.5 & $1.4-4.5$ \\
\hline GDM (without preeclampsia) & 0.9 & 41 & $35-47$ & 184 & $117-290$ & 22 & $18-27$ & 118 & $90-156$ \\
\hline Preeclampsia and GDM & 0.1 & 79 & $58-108$ & 430 & 204-905 & 52 & $34-79$ & 145 & $76-278$ \\
\hline
\end{tabular}

Data from pregnancies in 2,004-8 $(\mathrm{n}=226,832)$

GDM Gestational diabetes mellitus

* Excluding those receiving insulin only or oral antidiabetics only 
the proportion of women with GDM receiving drugs used to treat diabetes after giving birth, was very high.

Some doctors may prescribe insulin to breastfeeding women in order to control their blood glucose levels and reduce potential harm to the infants. We repeated the analyses excluding the first 6 months after giving birth. This exclusion had little impact on the results.

Other studies have documented the association between GDM and subsequent diabetes, but this is, to our knowledge, the largest and most comprehensive study.

Another study [19] found a positive predictive value of GDM in the MBRN, i.e. the proportion of women registered with GDM in MBRN having GDM, of $89 \%$ for births in 1998. However, GDM may be underreported in MBRN, and only the most serious cases of GDM may have been registered. If so, mothers with less severe GDM have been included in the reference group in our study. The group of mothers with GDM in this study will then, however, probably consist of women with severe GDM.

We had no information on the weight of the mothers, neither before, during nor after pregnancy. Obesity is one of the main risk factors for diabetes type 2. It is also associated with both preeclampsia and GDM. Hence, in a population with higher BMI, the association between GDM/preeclampsia and subsequent diabetes probably would have been stronger than observed here. Other potential confounders are family history of DM, socioeconomic status (SES), ethnicity, parity, folic acid use during pregnancy, smoking and twinning. We made adjustments for the parity of the mothers in the analyses. Rough smoking habits at the beginning and end of pregnancy was available for $79 \%$ of the women. In women not smoking neither at the beginning nor end of pregnancy, the results was about the same as for the entire study population. Use of folic acid during pregnancy is recorded in MBRN (yes/no); about $51 \%$ of the women used folic acid. Including this information in the analysis, did not change the results. The issue of twinning is complex and needs specific attention. However, in this study we chose to restrict the included pregnancies to those resulting in single births. The severity of GDM and when GDM was manifested was not recorded. Severity and timing of GDM may be related to the risk of getting diabetes later.

Disease codes were not included in NorPD before March 2009, so we could only exclude women having hypertension prior to pregnancy according to MBRN. About $1 \%$ of the women included, used medications prescribed to treat hypertension (among other conditions) prior to pregnancy. Excluding these women resulted in only small changes in the results.

Some women had more than one pregnancy during the study period. We chose to include only the first pregnancy for each woman in the study period. By doing this, we avoided to include the same event (being prescribed and dispensed antidiabetic drugs) several times and avoided dependencies within the data set.

Some diabetic women do not use drugs for diabetes. In the US, overall $16 \%$ of adults with diagnosed diabetes (type 1 or type 2 ), did not take insulin nor oral medication [5]. In our study, the indicated proportion of mothers developing diabetes after pregnancy may be underestimated. However, our results would be influenced only if the proportion of diabetics not receiving drugs differed according to whether they had GDM and/or preeclampsia.

Most studies on later risk of diabetes in mothers after pregnancies complicated with preeclampsia or GDM focus on the risk of type 2 diabetes [7-10]. In our study we could not separate the two main types of diabetes in data from NorPD. However, since women with type 1 diabetes before pregnancy were excluded, and this type of diabetes usually is diagnosed during childhood and adolescence, most of the women receiving antidiabetic drugs in our study probably had type 2 diabetes.

In a Norwegian hospital-based clinical study of women with GDM [24], 60\% developed diabetes within 16 years. In a Canadian cohort study, 19\% of women with GDM developed diabetes during the first 9 years [7]. According to Kjos and Buchanan, women with GDM have 17-63\% risk of nongestational diabetes within 5 to 16 years after pregnancy [25]. We estimated that in women having GDM, but not preeclampsia, $19 \%$ received drugs used to treat diabetes within 5 years after giving birth. In a Danish cohort study, Lykke et al. [14] found a threefold increased risk of diabetes type 2 after a mean follow-up of 15 years in women with preeclampsia. After a mean follow-up of 3.7 years, we found a threefold increased risk of receiving drugs used to treat diabetes.

In women without GDM and preeclampsia, the frequency of using drugs used to treat diabetes 5 years after giving birth was low. Women having pregnancies complicated with preeclampsia or GDM had an increased risk of later diabetes. The relative risk was high in women having GDM (and highest in women with both GDM and preeclampsia). A high proportion of mothers with GDM will need drugs to treat diabetes within 5 years after pregnancy. If the increase of GDM registered in MBRN in recent years is real, a further increase in diabetic women can be expected. Women with GDM should be a target group for interventions aimed at preventing type 2 diabetes.

Conflict of interest The authors declare that they have no conflict of interest.

Open Access This article is distributed under the terms of the Creative Commons Attribution Noncommercial License which permits any noncommercial use, distribution, and reproduction in any medium, provided the original author(s) and source are credited. 


\section{References}

1. Wild S, Roglic G, Green A, Sicree R, King H. Global prevalence of diabetes: estimates for the year 2000 and projections for 2030 . Diabetes Care. 2004;27:1047-53.

2. Roglic G, Unwin N, Bennett PH, Mathers C, Tuomilehto J, Nag $\mathrm{S}$, et al. The burden of mortality attributable to diabetes: realistic estimates for the year 2000. Diabetes Care. 2005;28:2130-5.

3. Buchanan TA, Xiang A, Kjos SL, Watanabe R. What is gestational diabetes? Diabetes Care. 2007;30(Suppl 2):S105-11.

4. WHO. Diabetes action now booklet. (2010).

5. Centers for Disease Control and Prevention. National diabetes fact sheet: general information and national estimates on diabetes in the United States, 2007. Atlanta, GA, US Department of Health and Human Services, Centers for Disease Control and Prevention (2008).

6. The Medical Birth Registry of Norway. Statistics. 2010. http://mfr-nesstaruibno/mfr/.

7. Feig DS, Zinman B, Wang X, Hux JE. Risk of development of diabetes mellitus after diagnosis of gestational diabetes. CMAJ. 2008;179:229-34.

8. Järvelä IY, Juutinen J, Koskela P, Hartikainen AL, Kulmala P, Knip M, et al. Gestational diabetes identifies women at risk for permanent type 1 and type 2 diabetes in fertile age: predictive role of autoantibodies. Diabetes Care. 2006;29:607-12.

9. Bellamy L, Casas JP, Hingorani AD, Williams D. Type 2 diabetes mellitus after gestational diabetes: a systematic review and metaanalysis. Lancet. 2009;373:1773-9.

10. Ben Haroush A, Yogev Y, Hod M. Epidemiology of gestational diabetes mellitus and its association with Type 2 diabetes. Diabet Med. 2004;21:103-13.

11. Metzger BE, Lowe LP, Dyer AR, Trimble ER, Chaovarindr U, Coustan DR, et al. Hyperglycemia and adverse pregnancy outcomes. N Engl J Med. 2008;358:1991-2002.

12. Yogev, Chen, Hod, Coustan, Oats, McIntyre, et al. The Hyperglycemia and Adverse Pregnancy Outcome (HAPO) Study Cooperative Research Group. Hyperglycemia and Adverse Pregnancy Outcome (HAPO) Study: preeclampsia. Am J Obstet Gynecol 2010; 202: 255.e1-7.

13. Callaway LK, Lawlor DA, O'Callaghan M, Williams GM, Najman JM, McIntyre HD. Diabetes mellitus in the 21 years after a pregnancy that was complicated by hypertension: findings from a prospective cohort study. Am J Obstet Gynecol. 2007;197:492-7.

14. Lykke JA, Langhoff-Roos J, Sibai BM, Funai EF, Triche EW, Paidas MJ. Hypertensive pregnancy disorders and subsequent cardiovascular morbidity and type 2 diabetes mellitus in the mother. Hypertension. 2009;53:944-51.

15. Engeland A, Bramness JG, Daltveit AK, Rønning M, Skurtveit S, Furu K. Prescription drug use among fathers and mothers before and during pregnancy. A population-based cohort study of 106, 000 pregnancies in Norway 2004-06. Br J Clin Pharmacol. 2008;69:653-60.

16. Furu K. Establishment of the nationwide Norwegian Prescription Database (NorPD) - new opportunities for research in pharmacoepidemiology in Norway. Nor J Epidemiol 2008; 18: 129-136. (http://www.ntnu.no/ojs/index.php/norepid/article/view/23).

17. WHO Collaborating Centre for Drug Statistics Methodology. WHO Collaborating Centre for Drug Statistics Methodology. 2010. http://www.whoccno/.

18. Irgens LM. The medical birth registry of Norway. Epidemiological research and surveillance throughout 30 years. Acta Obstet Gynecol Scand. 2000;79:435-9.

19. Stene LC, Eidem I, Vangen S, Joner G, Irgens LM, Moe N. The validity of the diabetes mellitus diagnosis in the Medical Birth Registry of Norway. Nor J Epidemiol 2007; 17: 165-174. (http:// www.ntnu.no/ojs/index.php/norepid/article/view/158).

20. The Norwegian Society of Gynecology and Obstretics. Clinical Guidelines in Obstetrics. 2010 http://www.legeforeningenno/id/ 1317710.

21. SPSS Inc. SPSS for Windows. Release 14.0.2. 22-11-2006.

22. StataCorp. Stata version 10.1. 2008. Texas, USA.

23. Engeland A, Bjørge T, Daltveit AK, Vollset SE, Furu K. Validation of disease registration in pregnant women in the Medical Birth Registry of Norway. Acta Obstetricia Gynecologica Scandinavica. 2009;88:1083-9.

24. Dale PO, Moe N, Jervell J. Pregnancy in diabetes. Tidsskr Nor Laegeforen. 1988;108:2968-70.

25. Kjos SL, Buchanan TA. Gestational diabetes mellitus. N Engl J Med. 1999;341:1749-56. 